\title{
AVALIAÇÃO DA PRODUTIVIDADE E VIGOR VEGETATIVO DE LINHAGENS DAS CULTIVARES CATUAÍ VERMELHO E CATUAÍ AMARELO (Coffea arabica L.) PLANTADAS INDIVIDUALMENTE E EM DIFERENTES COMBINAÇÕES ${ }^{1}$
}

\author{
Vegetative vigor and yield evaluations of coffee cultivars, "Catuai Vermelho" \\ and "Amarelo" (Coffea arabica L.) planted isolated and different combinations
}

\author{
Ângela Maria Nogueira ${ }^{2}$, Samuel Pereira de Carvalho ${ }^{3}$, Gabriel Ferreira Bartholo ${ }^{4}$, \\ Antônio Nazareno Guimarães Mendes ${ }^{3}$
}

\begin{abstract}
RESUMO
Instalou-se o experimento na Fazenda Experimental da EPAMIG, em São Sebastião do Paraíso, MG, com o objetivo de avaliar a característica de produtividade de linhagens das cultivares Catuaí Vermelho (IAC 44, IAC 81 e IAC 99) e Catuaí Amarelo (IAC 47, IAC 62 e IAC 86), no período de 1994 a 1999, plantadas isoladas e em diferentes combinações. O experimento foi instalado obedecendo ao delineamento experimental de blocos ao acaso, com três repetições, parcelas constituídas de seis covas, com uma planta em cada cova, adotando o sistema de manejo usualmente empregado na região, no espaçamento de 3,50 m entre linhas x 1,00 m entre covas. As linhagens foram agrupadas por cultivar, plantadas isoladas e em multilinhas nas proporções de 33\% e 50\%. As análises foram realizadas considerando a característica avaliada individualmente e empregando o teste de Duncan para comparação de médias. Em função dos resultados ficou evidenciado o efeito das combinações das diferentes linhagens das cultivares sobre a produtividade e vigor vegetativo. Linhagens das cultivares de Catuaí quando plantadas em multilinhas podem ser mais produtivas e vigorosas em comparação ao plantio individual. A cultivar Catuaí Vermelho IAC 44 em combinação com a IAC 81, e a combinação das linhagens IAC 81 com IAC 99 na proporção de 50\% de cada, verificaram uma interação positiva, pois apresentaram produtividade maior que quando plantadas isoladamente. Para a cultivar Catuaí Amarelo a melhor combinação foi da linhagem IAC 47 com IAC 62, também na proporção de $50 \%$. Com relação ao vigor vegetativo, pode-se considerar que não houve diferença entre as linhagens, e que a variação ocorrida pode ser atribuída a interação da linhagem com o ambiente em que estava sendo cultivada.
\end{abstract}

Termos para indexação: café, produção, Coffea arabica, combinação, isoladas.

\begin{abstract}
One experiment was conducted at EPAMIG`s experimental station at São Sebastião do Paraíso, MG, to evaluate yield characteristics and vegetative vigor, of "Catuaí Vermelho" (IAC 44, IAC 81, and IAC 99), "Catuai Amarelo" (IAC 47, IAC 62, and IAC 86), coffee lineage cultivars from 1994 to 1999, planted isolated and in different combinations. An experimental randomized blocks design was used, with 3 replications 14 lineages combination as treatment, each plot with six plants, spaced 3.5 by $1.0 \mathrm{~m}$, respectively among interrows and lines. Lineages cultivars were grouped and planted isolated and in multi-lines at proposition of $33 \%$ and $50 \%$. Characteristics were evaluated individually, and averages compared by Duncan test. Coffee cultivars combinations planted in multi-lines showed greater yield and greater vigor plants as compared to isolated planting. "Catuaí Vermelho" IAC 44 cultivar in combination with the IAC 81 and the lineage combinations of the IAC 81 and IAC 99 at $50 \%$ proportion of each, showed positive interaction by the fact of to present greater yield than when planted isolated. Take in account lineages of "Catuaí Amarelo", better combination was found with IAC 47 by IAC 62 at 50\% proportion. There were no differences in vegetative vigor among lineages; some differences occurred is due to environment interactions with lineage.
\end{abstract}

Index terms: Coffee yield, Coffea arabica, and multi-lines, isolated.

(Recebido para publicação em 8 de abril de 2003 e aprovado em 16 de julho de 2004)

\section{INTRODUÇÃO}

As lavouras cafeeiras do Brasil são formadas em sua grande maioria, por cultivares da espécie Coffea arabica, responsáveis por um produto de boa qualidade e de maior aceitação pelo mercado consumidor.

Originalmente, os cafezais foram formados a partir de plantas derivadas da variedade "arábica",

1. Artigo extraído da Tese de Doutorado apresentada à Universidade Federal de Lavras/UFLA, Caixa Postal 3037 - 37.200-000 - Lavras, MG.

2. Doutoranda em Agronomia/Fitotecnia.

3. Professor Adjunto Departamento de Agricultura/UFLA.

4. Pesquisador da EPAMIG. 
a primeira a ser introduzida no Brasil. Gradativamente, foram sendo diversificados pela introdução de novas cultivares como Bourbon Vermelho e Sumatra, ou por material originado no Brasil, como amarelo de Botucatu, maragogipe, caturra ou bourbon amarelo. $\mathrm{O}$ "caturra”, tanto o amarelo como o vermelho, apesar de produtivo e de ter a vantagem do porte pequeno, não foi intensamente cultivado por não se adaptar bem nas condições ambientais das diversas regiões cafeeiras.

Em 1943, o Instituto Agronômico de Campinas, iniciou a seleção dentro de uma população de cafeeiros de plantas rústicas, vigorosas, de elevada produção, resultante da hibridação natural ocorrida entre as cultivares Bourbon Vermelho e Sumatra. As seleções efetuadas deram origem à cultivar Mundo Novo, uma das mais produtivas e que rapidamente se expandiu por todas as regiões do Brasil (MARTINS et al., 1992).

Em 1949, foram realizadas as primeiras hibridações entre plantas selecionadas de Caturra Amarelo e de Mundo Novo, no intuito de associar a rusticidade e a produtividade de Mundo Novo ao porte reduzido de Caturra, característica de grande interesse econômico, por facilitar os tratos da lavoura e a colheita do produto. Dessas hibridações e das seleções efetuadas nas gerações subseqüentes, resultaram as cultivares Catuaí Amarelo e Catuaí Vermelho, de porte reduzido, alto vigor vegetativo e produção (Carvalho e Mônaco, 1972).

Jensen (1952) propôs um sistema de estabelecimento de multilinha, formado com mistura de linhas puras superiores e compatíveis, uniformes na aparência, ciclo produtivo, altura de plantas, hábito de crescimento, coloração, tipo e tamanho dos grãos, ou seja, quando a uniformidade das plantas ou do produto for necessária.

Indicações para o uso de multilinhas para a resistência a doenças, têm sido feitas por Jensen (1952) e Browning e Frey (1969) para o cultivo de aveia, Borlaug (1965) para o trigo, Suneson (1960) para a cevada, Shands et al. (1964) para o feijão e vários outros autores para diferentes espécies de interesse. Jensen (1965) ressalta que multilinhas de aveia produzem bem e de modo mais estável.

Outra questão a ser considerada é, que, se uma multilinha não for constituída por linhas isogênicas ela pode ser alterada com o decorrer do tempo em razão da seleção natural, e a sobrevivência de cada genótipo numa mistura depende da capacidade produtiva e da proporção de sementes de cada genótipo atingirem a fase de maturação e deixar descendentes. Esses fatores são resultantes de uma ação combinada de outros fatores, mas em sua maioria ainda são desconhecidos (BUENO et al., 1999).
Em razão das linhagens estabelecidas das cultivares Catuaí Amarelo e Catuaí Vermelho apresentarem base genética muito próxima e as características fenotípicas não possuírem diferenças marcantes, foi idealizado este trabalho, com o objetivo de avaliar a produção e o vigor vegetativo de linhagens das cultivares de Catuaí Vermelho e Catuaí Amarelo, plantadas isoladas e em diferentes combinações, e verificar se o uso de multilinhas pode ser aplicado com eficiência na cafeicultura.

\section{MATERIAL E MÉTODOS}

$\mathrm{O}$ experimento foi instalado em um Latossolo Vermelho distroférrico (LVd), na Fazenda Experimental de EPAMIG em São Sebastião do Paraíso, MG, numa altitude de $890 \mathrm{~m}$, latitude $20^{\circ} 55^{\prime} \mathrm{S}$ e longitude $46^{\circ} 55^{\prime} \mathrm{W}$, com precipitação pluvial média de $1470 \mathrm{~mm}$ distribuídas de outubro a abril, e temperatura média anual de $20,8^{\circ} \mathrm{C}$.

$\mathrm{O}$ material estudado compreende três linhagens da cultivar Catuaí Vermelho, IAC 44, IAC 81 e IAC 99 e três da cultivar 'Catuaí Amarelo', IAC 47, IAC 62 e IAC 86. As linhagens foram agrupadas por cultivar e plantadas isoladas e em misturas de mudas nas proporções de $50 \%$ e $33 \%$, respectivamente.

Os tratamentos foram os seguintes: $\mathrm{T}_{1}=$ 'Catuaí Vermelho' IAC 44; $\mathrm{T}_{2}=$ 'Catuaí Vermelho' IAC 81; $\mathrm{T}_{3}=$ 'Catuaí Vermelho' IAC 99; $\mathrm{T}_{4}=$ IAC $44+$ IAC 81; $\mathrm{T}_{5}=$ IAC 44 + IAC 99; $\mathrm{T}_{6}=$ IAC 81 + IAC 99; $\mathrm{T}_{7}=$ IAC 44 + IAC 81 + IAC 99; $\mathrm{T}_{8}=$ 'Catuaí Amarelo' IAC 47; $\mathrm{T}_{9}=$ 'Catuaí Amarelo' IAC 62; $\mathrm{T}_{10}=$ 'Catuaí Amarelo' IAC 86; $\mathrm{T}_{11}=$ IAC $47+$ IAC 62; $\mathrm{T}_{12}=$ IAC $47+$ IAC 86; $\mathrm{T}_{13}$ $=$ IAC $62+$ IAC 86; T $_{14}=$ IAC $47+$ IAC $62+$ IAC 86.

O delineamento experimental utilizado foi em blocos ao acaso, com três repetições, contendo seis plantas por parcela, sem bordaduras laterais; para a coleta de dados, toda a parcela foi considerada útil. Foi considerado o teste de Duncan a $1 \%$ de probabilidade para comparação de médias.

Foi avaliada a produtividade de grãos, para estudo das linhagens. As colheitas foram realizadas na primeira quinzena do mês de junho, avaliadas em quilos de café cerejas por parcela, das quais foram retiradas amostras de $2 \mathrm{~kg} /$ parcela, que foram acondicionadas em sacos de plástico telado para secagem em terreiro até atingir o ponto de umidade em $11,5 \%$. Posteriormente foram beneficiadas em um descascador de amostras, calculando o rendimento de café beneficiado e transformando a produção para sacas de café beneficiado por hectare.

$\mathrm{O}$ vigor vegetativo foi avaliado anualmente por ocasião da colheita, atribuindo-se notas arbitrárias numa escala de 1 a 10, em função do desenvolvimento vegetativo das plantas, sendo a nota 1 atribuída às plantas pouco vigorosas e 10 àquelas com ótimo desenvolvimento vegetativo. 


\section{RESULTADOS E DISCUSSÃO}

A produtividade em sacos de $60 \mathrm{Kg}$ /ha de café beneficiado, foi influenciada significativamente pelos tratamentos, anos de colheita e pela interação entre esses fatores. As médias da produtividade, considerando os seis anos, foram influenciadas pelos tratamentos, como evidenciado pela análise de variância apresentada na Tabela 1.

O efeito dos tratamentos sobre a produtividade em relação aos anos de colheita foi destacado pelo desdobramento da interação, mostrando que os anos de 1996 e 1998 foram os que apresentaram diferenças entre os tratamentos, como pode ser visto na Tabela 2.

TABELA 1 - Resumo da análise de variância considerando a média dos seis anos, para a produtividade das linhagens de Catuaí Vermelho e Catuaí Amarelo, plantadas individualmente ou em diferentes combinações, em São Sebastião do Paraíso, MG. 2003.

\begin{tabular}{lrc}
\hline \multicolumn{1}{c}{ FV } & GL & QM \\
\hline Repetição & 2 & 28,09 \\
Tratamento & 13 & $147,49^{* *}$ \\
Erro(a) & 26 & 20,36 \\
Ano & 5 & $3088,64^{* *}$ \\
Erro (b) & 10 & 260,16 \\
Tratamento x Ano & 65 & $58,24^{*}$ \\
Erro (c) & 130 & 38,66 \\
\hline Total & 251 & \\
\hline CV (a)\% 23,75 & & \\
CV (b)\% 84,92 & & \\
CV (c)\% 32,74 & & \\
\hline
\end{tabular}

** Significativo a $1 \%$ de probabilidade

TABELA 2 - Análise de variância para o desdobramento da interação tratamentos dentro de cada ano, considerando a produtividade das linhagens de Catuaí Vermelho e Catuaí Amarelo, plantadas individualmente ou em diferentes combinações, em São Sebastião do Paraíso, MG. 2003.

\begin{tabular}{lcc}
\hline \multicolumn{1}{c}{ FV } & GL & QM \\
\hline Tratamento dentro do ano -1994 & 13 & 3,79 \\
Tratamento dentro do ano - 1995 & 13 & 35,06 \\
Tratamento dentro do ano -1996 & 13 & $118,56^{* *}$ \\
Tratamento dentro do ano - 1996 & 13 & 25,61 \\
Tratamento dentro do ano - 1998 & 13 & $209,65^{* *}$ \\
Tratamento dentro do ano - 1999 & 13 & 45,99 \\
Erro & 150 & 35,61 \\
\hline CV (\%) 32,74 & & \\
\hline
\end{tabular}


Na Tabela 3, observa-se que no ano de 1996, o tratamento quatro, composto pela combinação da mistura das linhagens de Catuaí Vermelho (IAC 44 + IAC 81), na proporção de $50 \%$ de cada, apresentou uma produtividade de 50,66 sacas de $60 \mathrm{~kg}$ de café beneficiado, embora estatisticamente igual aos tratamentos 6 (IAC 81 + IAC 99) e 9 (IAC 62), mantendo a mesma tendência no ano de 1998. Ano em que os tratamentos 2 (IAC 81), 5 (IAC 44 + IAC 99), 6 (IAC 81 + IAC 99), 9 (IAC 62), 11 (IAC 47 + IAC 62) e 14 (IAC 47 + IAC 62 + IAC 86) também se mantiveram como os mais produtivos.

Procedeu-se à comparação de médias utilizando o teste de Duncan a $1 \%$ de probabilidade, como apresentada na Tabela 4.

Constatou-se que a mistura das linhagens de Catuaí Vermelho (IAC 44 + IAC 81) na proporção de 50\% de cada uma, apresenta-se como a mais produtiva e pelo resultado do teste de médias é igual estatisticamente aos tratamentos 11 (IAC 47 + IAC 62), 6 (IAC $81+$ IAC 99), 2 (IAC 81) e 9 (IAC 62), embora, estes não diferindo significativamente dos tratamentos 3 (IAC 99), 5 (IAC 44 + IAC 99), 13 (IAC 62 +IAC 86), 14 (IAC 47 + IAC 62 + IAC 86) e 8 (IAC 47), que tiveram com- portamento semelhante, com nível de produtividade variando de 21,28 sacas com $60 \mathrm{Kg}$ de café beneficiado por hectare até 26,80 sacas. Entretanto, esse grupo de tratamentos é estatisticamente igual aos tratamentos que apresentaram os menores níveis de produtividade, que foram os tratamentos 12 (IAC 47 +IAC 86), 7 (IAC 44 + IAC 81 + IAC 99), 1 (IAC 44) e 10 (IAC 86).

Neste trabalho ficou evidente uma pequena vantagem da mistura de linhagens, entretanto, devido à base genética ser muito estreita, pode-se pressupor que as variações ocorridas na produtividade sejam em função do ambiente em que foram cultivadas. Assim, as misturas não mostraram eficiência para melhorar a produtividade das cultivares, mas, a amplitude de adaptabilidade de cada linhagem, fato que se torna importante na escolha do material a ser cultivado em cada região produtora de café.

Analisando o comportamento das cultivares ao longo dos seis anos, para a característica vigor vegetativo, pode-se observar pela análise de variância (Tabela 5) , que houve diferenças significativas nos tratamentos em teste, no decorrer dos anos em que o experimento foi desenvolvido.

TABELA 3 - Produtividade de café beneficiado em sacos de $60 \mathrm{~kg} / \mathrm{ha}$, considerando os anos de 1996 e 1998, das linhagens de Catuaí Vermelho e Catuaí Amarelo, plantadas individualmente ou em diferentes combinações, em São Sebastião do Paraíso, MG. 2003.

\begin{tabular}{clcc}
\hline $\mathbf{N}^{\mathbf{1}}$ & Tratamentos & \multicolumn{2}{c}{ Anos } \\
\cline { 3 - 4 } & & $\mathbf{1 9 9 6}$ & $\mathbf{1 9 9 8}$ \\
\hline 1 & (IAC 44) & $23,68 \mathrm{~d}$ & $28,00 \mathrm{cdef}$ \\
2 & (IAC 81) & $39,71 \mathrm{~b}$ & $41,73 \mathrm{abcd}$ \\
3 & (IAC 99) & $36,18 \mathrm{bcd}$ & $36,33 \mathrm{bcde}$ \\
4 & (IAC 44+ IAC 81) & $50,66 \mathrm{a}$ & $53,67 \mathrm{a}$ \\
5 & (IAC44 + IAC99) & $37,69 \mathrm{bc}$ & $44,83 \mathrm{abc}$ \\
6 & (IAC 81 + IAC 99) & $40,90 \mathrm{ab}$ & $41,33 \mathrm{abcd}$ \\
7 & (IAC 44 + IAC 81 + IAC 99) & $27,63 \mathrm{bcd}$ & $26,89 \mathrm{def}$ \\
8 & (IAC 47) & $26,02 \mathrm{~cd}$ & $30,38 \mathrm{bcd}$ \\
9 & (IAC 62) & $39,71 \mathrm{ab}$ & $47,52 \mathrm{ab}$ \\
10 & (IAC 86) & $24,36 \mathrm{~cd}$ & $19,31 \mathrm{f}$ \\
11 & (IAC 47 + IAC 62) & $36,50 \mathrm{bcd}$ & $39,00 \mathrm{abcde}$ \\
12 & (IAC 47 + IAC 86) & $30,46 \mathrm{bcd}$ & $24,78 \mathrm{def}$ \\
13 & (IAC 62 + IAC86) & $32,64 \mathrm{bcd}$ & $27,85 \mathrm{cdef}$ \\
14 & (IAC 47+ IAC 62 + IAC 86) & $34,07 \mathrm{bcd}$ & $39,83 \mathrm{abcde}$ \\
\hline
\end{tabular}

Médias seguidas da mesma letra, não diferem entre si, pelo teste de Duncan a $1 \%$ de probabilidade. 
TABELA 4 - Comparação das médias dos 6 anos considerando a produtividade para as linhagens de Catuaí Vermelho e Catuaí Amarelo, plantadas isoladas e em diferentes combinações em São Sebastião do Paraíso. UFLA, Lavras, 2003.

\begin{tabular}{|c|c|c|}
\hline Número & Tratamentos & Médias \\
\hline 4 & (IAC 44 + IAC 81) & $27,88 \mathrm{a}$ \\
\hline 11 & (IAC 47 + IAC 62) & $26,80 \mathrm{ab}$ \\
\hline 6 & (IAC $81+$ IAC 99) & $26,35 \mathrm{ab}$ \\
\hline 2 & (IAC 81) & $26,29 \mathrm{ab}$ \\
\hline 9 & (IAC 62) & 25,44 abc \\
\hline 3 & (IAC 99) & 22,11 bcd \\
\hline 5 & (IAC $44+$ IAC 99) & 21,99 bcd \\
\hline 13 & (IAC $62+$ IAC 86) & 21,97 bcd \\
\hline 14 & $($ IAC $47+$ IAC $62+$ IAC 86) & 21,39 bcd \\
\hline 8 & (IAC 47) & 21,28 bcd \\
\hline 12 & (IAC $47+$ IAC 86) & $20,26 \mathrm{~cd}$ \\
\hline 7 & (IAC 44 + IAC 81 + IAC 99) & $18,94 \quad d$ \\
\hline 1 & (IAC 44) & 18,18 \\
\hline 10 & (IAC 86) & $17,43 \quad d$ \\
\hline
\end{tabular}

Médias seguidas da mesma letra, não diferem entre si, pelo teste de Duncan a $1 \%$ de probabilidade.

TABELA 5 - Resumo da Análise de Variância considerando a média dos 6 anos para o vigor vegetativo das linhagens Catuaí Vermelho e Catuaí Amarelo, plantadas isoladas e em diferentes combinações, em São Sebastião do Paraíso. UFLA, Lavras, 2003.

\begin{tabular}{lcc}
\hline & & QM \\
\cline { 3 - 3 } \multicolumn{1}{c}{ FV } & GL & Vigor vegetativo \\
\hline Repetição & 2 & 0,07 \\
Tratamento & 13 & $0,69 * *$ \\
Erro & 26 & 0,11 \\
Total & 41 & 1,56 \\
\hline
\end{tabular}

** Significativo a $1 \%$ de probabilidade

Com base nos resultados apresentados na Tabela 6, pode-se deduzir que as variações ocorridas nos tratamentos determinam que as linhagens IAC 62 (tratamento 9) e IAC 81 (tratamento 2) de Catuaí Amarelo e Catuaí Vermelho, respectivamente, não são diferentes estatisticamente dos tratamentos 8 (IAC 47), 11 (IAC $47+$ IAC 62), 4 (IAC 44 + IAC 81), 12 (IAC 47 + IAC 86), 13(IAC 62 + IAC 86), 6 (IAC 81 + IAC 99), 5 (IAC 44 + IAC 99), 14 IAC 47 + IAC 62 + IAC 86), 3 (IAC 99) e 10 (IAC 86), o que demonstra a uniformidade do material em estudo para essa característica. Tendo em vista que não há diferença genotípica, as variações ocorridas podem ser atribuídas a influência do ambiente, visto que, entre as linhagens algumas têm adaptabilidade específica para outros ambientes, como a linhagem de Catuaí Vermelho IAC 44, que, quando cultivada de forma isolada, apresentou o menor vigor e, entretanto, não diferiu estatisticamente dos tratamentos 13 (IAC $62+$ IAC 86), 6 (IAC 81 + IAC 99), 5 (IAC 44 + IAC 99), 14 (IAC 47 + IAC 62 + IAC 86), 3 (IAC 99), 10 (IAC 86) e 7 (IAC 44 + IAC 81 + IAC 99). Embora existam diferenças significativas, o vigor de todas as linhagens, sejam isoladas ou combinadas, foi considerado de médio a alto de acordo com o Índice de Avaliação Visual (IAV). 
TABELA 6 - Comparação das médias dos 6 anos considerando o vigor vegetativo para as linhagens de Catuaí Vermelho e Catuaí Amarelo, plantadas isoladas e em diferentes combinações em São Sebastião do Paraíso. UFLA, Lavras, 2003

\begin{tabular}{rlc}
\hline $\mathbf{N}^{\mathbf{0}}$ & \multicolumn{1}{c}{ Tratamentos } & Médias \\
\hline 9 & (IAC 62) & $7,58 \mathrm{a}$ \\
2 & (IAC 81) & $7,58 \mathrm{a}$ \\
8 & (IAC 47 & $7,47 \mathrm{ab}$ \\
11 & (IAC 47 + IAC 62) & $7,41 \mathrm{ab}$ \\
4 & (IAC 44 + IAC 81) & $7,36 \mathrm{ab}$ \\
12 & (IAC 47 + IAC 86) & $7,25 \mathrm{ab}$ \\
13 & (IAC 62 + IAC 86) & $7,16 \mathrm{abc}$ \\
6 & (IAC 81 + IAC 99) & $7,08 \mathrm{abc}$ \\
5 & (IAC 44 + IAC 99) & $6,80 \mathrm{abc}$ \\
14 & (IAC 47 + IAC 62 + IAC 86) & $6,77 \mathrm{abc}$ \\
3 & (IAC 99) & $6,66 \mathrm{abc}$ \\
10 & (IAC 86) & $6,63 \mathrm{abc}$ \\
7 & (IAC 44 + IAC 81 + IAC 99) & 6,36 bc \\
1 & (IAC 44) & $6,08 \quad \mathrm{c}$ \\
\hline
\end{tabular}

Médias seguidas da mesma letra, não diferem entre si, pelo teste de Duncan a 1\% de probabilidade.

\section{CONCLUSÃO}

O efeito das combinações de linhagens cultivares Catuaí Amarelo e Catuaí Vermelho sobre a produtividade, apresentaram variações quando plantadas em combinações na proporção de $50 \%$, entretanto, quando observada a produtividade das linhagens cultivadas individualmente, a maioria delas esteve no grupamento de menor produtividade.

Linhagens das cultivares de Catuaí Vermelho ou de Catuaí Amarelo, quando plantadas em multilinhas podem ser mais produtivas e vigorosas em comparação ao plantio individual.

A cultivar Catuaí Vermelho IAC 44 em combinação com a IAC 81, a combinação da linhagem IAC 81 com IAC 99 na proporção de 50\% de cada, mostraram uma interação positiva, pois, apresentaram produtividade maior que quando plantadas isoladamente.

Para a cultivar Catuaí Amarelo a melhor combinação foi da linhagem IAC 47 com IAC 62, também na proporção de $50 \%$. Entretanto em se tratando de materiais cuja base genética é muito próxima, e a tradição de produção de sementes é de forma individualizada por linhagem, a mistura das linhagens não se justifica, visto que o acréscimo na produção foi muito pequeno.

Considerando o vigor vegetativo, pode-se dizer que, não há diferenças entre as linhagens, sendo que a variação ocorrida é atribuída à interação da linhagem com o ambiente em que estava sendo cultivada.

Os resultados obtidos são considerados para o ecossistema da região de São Sebastião do Paraíso. Para outras regiões cafeeiras de Minas Gerais, a extrapolação deve ser feita com base nos resultados dos estudos de adaptabilidade desses materiais.

\section{REFERÊNCIAS BIBLIOGRÁFICAS}

BORLAUG, N. E. Wheat, rust and people. Phytopathology, Saint Paul, v. 55, p. 1098, 1965.

BROWNING, J. A.; FREY, K. J. Multiline cultivars as a mean of disease control. Annual Review of Phytopathology, Saint Paul, v. 7, p. 355-382, 1969.

BUENO, L. C. S.; MENDES, A. N. G.; CARVALHO, S. P. Melhoramento genético de plantas. Lavras: UFLA/FAEPE, 1999. 
CARVALHO, A.; MÔNACO, L. C. Transferência do fator caturra para o cultivar Mundo Novo de Coffea arabica. Bragantia, Campinas, v. 31, n. 31, p. 99379, 1972.

JENSEN, N. F. Intra-varietal diversification in oat breeding. Agronomy Journal, Madison, v. 44, p. 3034, 1952.

JENSEN, N. F. Multiline superiority in cereais. Crop Science, Madison, v. 5, p. 5656-568, 1965.
MARTINS, A. L. M. et al. Avaliação de progênies de cafés 'Catuai Amarelo' e 'Catuaí Vermelho" na região de Pindorama (SP). Bragantia, Campinas, v. 51, n. 1, p. 31-38, 1992.

SHANDS, H.; VIEIRA, C.; ZAUMEYER, W. J. Observations on dry bean diseases in Brazil. Plant Disease Reporter, Washington, v. 48, p. 784-787, 1964.

SUNESON, C. A. Genetic diversity: a protectionagainst plant disease and insects. Agronomy Journal, Madison, v. 52, p. 319-321, 1960. 